\title{
Die Schönheit als Analogie zur Liebe? Darstellung der Liebe im Roman Hugo. Tragödie eines Knaben
}

\author{
Anežka Klimentová
}

In meinem Beitrag befasse ich mich mit den Konzepten der Liebe und der Schönheit. Ziel ist es, die Konstruktion von diesen Konzepten in Winders episodischem Roman Hugo. Tragödie eines Knaben (1924) zu erarbeiten. Ich gehe folgenden Fragen nach: Wie werden diese Konzepte im Roman präsentiert und zugleich gebrochen? Welche Rolle spielt die zeitgenössische (christliche) Moral und welche Rolle spielen die Frauenfiguren im Roman? Im Roman Hugo sucht Winder die Antwort auf die Frage, wie die Beziehung zwischen Liebesgefühl und körperlicher Anziehungskraft beschaffen ist bzw. ob man Schönheit als Bedingung für die Entwicklung eines Liebesgefühls wahrnehmen kann. Diese Frage wird dem Leser insbesondere durch die Figur Hugos vermittelt.

Die Handlung folgt drei Lebensetappen von Hugo Bandler, einem jüdischen Knaben. Während seines Knabenalters lernt Hugo verschiedene Formen der zwischenmenschlichen Beziehungen kennen, die aus der Sicht der zeitgenössischen Moral zweifellos als Perversität beurteilt würden. Als Beispiel dieser ,Perversität‘ kann man die Figur des Turnlehrers Pravda anführen, dem Hugo als Schüler auf dem Gymnasium begegnet. Pravda versammelt eine ausgewählte Gruppe von hübschen und unterwürfigen Schülern, zu denen er persönliche Beziehungen aufnimmt. Hugo, ein nicht so hübscher jüdischer Knabe, wird von Pravda ständig bloßgestellt. Die Interesselosigkeit Pravdas zwingt Hugo, sich zu bemühen, ständig die Aufmerksamkeit Pravdas zu gewinnen. Gleichzeitig wird Hugo immer neugieriger, was der Turnlehrer mit den Schülern in seinem Haus eigentlich unternimmt. Deshalb folgt er heimlich seinen Mitschülern, die das Haus des Turnlehrers besuchen, um das Geheimnis seines Lehrers zu entdecken. Die Tatsache, dass Pravda mit den Schülern in intimen Liebesverhältnissen steht, schockiert Hugo. Er entscheidet sich, sich an Pravda zu rächen. Aus Angst vor einem Skandal, der für ihn wahrscheinlich im Gefängnis enden würde (in der Tschechoslowakei wurde Homosexualität bis 1961 als Straftat beurteilt), beschließt Pravda, aus der Stadt zu fliehen (vgl. Winder 2012a: 20-37).

Diese Erfahrungen beeinflussen Hugo zutiefst. Im Bewusstsein des Kindes wird physische Anziehungskraft zum Synonym für Liebe: 
„Hugo ging gedankenvoll nach Hause. Er sagte es hundertmal: Er [Turnlehrer Pravda, AK] hat mir gedemütigt! Die Eltern saßen bereits beim Essen, er ignorierte es, ging in sein Zimmer, starrte sein Spiegelbild an. Ich bin häßlich! keuchte er, häßlich, häßlich! [...] Er lief ins Speisezimmer, aß die Suppe und sagte unvermittelt: ,Mutter, bin ich bestimmt nicht bucklig? Sag mir die Wahrheit!' ,Dummer Bub, ärgerte sich die Mutter, ,warum sollst du bucklig sein? In unserer Familie ist niemand bucklig. 'Bucklig bist du nicht, aber blöd, ' lachte der Vater. ,Das macht nichts, 'sagte Hugo ruhig, ,blöd will ich gern sein, daraus mach ich mir nichts, aber bucklig will ich nicht sein! Und häßlich will ich auch nicht sein!'“ (Winder 2012: 24-25)

Hugo entwickelt unbewusst eine Abneigung gegen hässliche Frauen, er verachtet sie sogar. Während seines Aufenthalts in der Stadt trifft Hugo Frau König, eine Hausfrau, die schon verwitwet ist und die in Hugos Mitbewohner Heß platonisch verliebt ist. Hugo beobachtet das geringschätzige und grobe Verhalten von Heß nicht nur gegenüber Frau König, sondern auch gegenüber anderen Frauen. Hugo ist zerrüttet. Er fühlt die moralische Pflicht, König zu helfen, in der Tiefe seiner Seele wird er aber von der einfachen und hässlichen Frau angeekelt (vgl. Winder 2012a: 38-57). Kurt Krolop schreibt:

„Die Bedeutung des Assoziationskomplexes ,Geheimnis‘, der schon für den ersten Teil konstitutiv gewesen war, nimmt in den beiden weiteren Teilen noch zu. Bei dem sechzehnjährigen Gymnasiasten, der ,eins der armseligen Bordellmädchen hie und da aufsuchte', ist nicht mehr die Sexualität als solche ein Geheimnis, sondern ihr Platz in einer Welt, deren ,Moral“ nach den Worten Bertrand Russels darin besteht, ,das eigene Triebleben zu nehmen und in das der anderen einzugreifen'; eine Moral, deren Sexualtabus von der Erziehung eingepflanzt, natürlich in Hugo Bandler weiterwirken und in ihm einen mörderischen und selbstmörderischen Kampf um die Identität von Schönheit und Reinheit auslöst, in dem er schließlich von Schmutz und Häßlichkeit überwältigt wird.“ (Winder 2012a: 187)

Hugo zieht aus Frau Königs Haus ins Haus der reichen Bürger, Herr und Frau Szell, um. Hier findet er allerdings auch keine Ruhe. Frau Szell hat eine Vorliebe für junge, attraktive Knaben, die von ihrem Mann ins Haus gebracht werden. Und umgekehrt: Frau Szell stellt junge, schöne Mädchen als Dienstmädchen an, die im Bett von Herrn Szell enden. Die Vorstellung, dass er nur ein Spielzeug einer alten frivolen Dame ist, erschreckt Hugo. Er überlegt sogar, Frau Szell zu ermorden (vgl. Winder 2012a: 5889).

Im Roman Hugo. Tragödie eines Knaben findet der Leser drei Typen von Liebesbeziehungen: Homosexualität, fast sklavische Besessenheit und ein Verhältnis zwischen einer älteren Dame und einem jüngeren Mann (bzw. umgekehrt). Alle diese Formen werden von der Gesellschaft als stark unerwünscht bewertet.

Die Absicht von Winder ist es aber nicht pornographische Motive auszuschöpfen, sondern tabuisierte Themen wie Homosexualität und Misshandlung von Frauen zur Sprache zu bringen. Kurt Krolop schreibt: „Wenn die zeitgenössische Kritik gelegentlich den 
Eindruck entstehen lässt, Turnlehrer Pravda sei ,nichts Anderes als eine Homosexualitäts-Novelle‘, dann reduziert sie das Erzählte auf das stoffliche Substrat eines Motivs, ohne dessen Funktion für die Erzählung zu berücksichtigen.“ (Krolop 2015: 177)

Was die Frage von Frauenmisshandlung betrifft, nimmt Winder eine progressive Position ein. Die Frauen werden von Winder nicht als einfache Objekte der Lust betrachtet, sondern als Opfer bzw. Produkte der Misshandlung der Männer. Es handelt sich also um eine scharfe Kritik an Männern, die Frauen nur als Sklavinnen oder billige Kokotten wahrnehmen. Frau König hat ihren Mann verloren. Aus Angst vor Einsamkeit sucht sie einen - beliebigen - Mann, der für sie ihren verstorbenen Ehemann ersetzen könnte. Die Vorliebe von Frau Szell für die jungen Knaben ist eigentlich eine Folge ihrer Ehekrise. Das Anliegen des Romans war nicht, Frauen zu demütigen, sondern sich für Frauen einzusetzen. Die Frauen werden also im Roman nicht als die moralisch niedrigen, sondern als leidende Personen dargestellt, die viel erfahren haben und die von den Männern misshandelt wurden.

Abschließend möchte ich auf die Frage eingehen, ob menschliche Hässlichkeit ihren Sinn und Zweck hat. Winder bietet uns zwei mögliche Lösungen: eine moralische und eine theologische. Die theologische Lösung wird in der Geschichte Legende vom hässlichen Menschen deutlich. Die Hauptfigur, Sebastian Laudon, diskutiert mit einem Bettler, ob die Schönheit von Gott gegeben sei. Auf seine Frage, ob Gott ihn durch Hässlichkeit bestraft habe, erwidert der Bettelbruder: „Du mußt es aushalten, denn gerade das soll deine Strafe sein, daß du erst nach fünfzehn Jahren heimkehren darfst." (Winder 2012b: 20).

Der Bettler erklärt die Hässlichkeit in theologischer Hinsicht. Du hast etwas Schlechtes gemacht, du hast deine Familie für fünfzehn Jahre verlassen, deshalb musst du deine Hässlichkeit als göttlichen Zorn ertragen.

Der moralische Schwerpunkt liegt in der Beschreibung und Enttabuisierung von Sexualität bzw. Homosexualität als eines natürlichen Bestandteiles des menschlichen Lebens. Homoerotische Beziehungen wurden als etwas Skandalöses, Abnormales, sogar Strafbares wahrgenommen. Winder strebt nach der Veränderung der gesellschaftlichen Konventionen, denn er stellt sich gegen die Vorstellung, dass die Frau der Besitz ihres Mannes und dass Homosexualität unakzeptabel sei. Nichtsdestoweniger bemerkt Kurt Krolop, dass das Urteil Hugos über Pravda ganz andere Wurzeln haben kann:

\footnotetext{
„Nicht ,Sittenrichterei und verschmähte Liebe“ sind die Hauptmotive für das Endurteil des Elfjährigen; es handelt sich weder primär um die Verurteilung eines ,Homosexuellen` durch den ,Puritanismus des Sittlichkeitsfanatikers‘ noch um den Racheakt eines Eifersüchtigen, sondern vor allem um die Verurteilung des Selbstverrates der Schönheit durch einen ,Schönheitsfanatiker‘, der Selbstprofanation eines Idols durch dessen Verehrer.” (Krolop 2015: 177)
}

In der Geschichte Hugos kann man zwei Hauptperspektiven betrachten, die der Autor gegenüberstellt. Es handelt sich um a) die Perspektive des Vaters von Hugo, der die 
Hässlichkeit (und vor allem die Hässlichkeit von Frauen) als ein „Malheur“ beurteilt (Winder 2012a: 25) und b) die Position von dem verwirrten und leicht manipulierbaren Hugo, der, ohne es zu bemerken, die Verhaltensmuster seines Vaters übernimmt. Seine Jagd nach der vollkommenen Liebe zu einer vollkommenen Dame bleibt erfolglos. „Alles oder nichts. Ich werde nur eine lieben, die mich umwirft.“ (Winder 2012a: 84)

\section{Quellen und Literatur}

Winder, Ludwig (2012a): Hugo. Tragödie eines Knaben. In: ders.: Hugo. Tragödie eines Knaben und andere Erzählungen (2. unveränd. Aufl.). Hamburg: Igel, S. 20-89.

Winder, Ludwig (2012b): Legende vom hässlichen Menschen. In: ders.: Hugo - Tragödie eines Knaben und andere Erzählungen (2. unveränd. Aufl.). Hamburg: Igel, S. 7-19.

Krolop, Kurt (2015): Ludwig Winder. Sein Leben und sein erzählerisches Frühwerk. Ein Beitrag zur Geschichte der Prager deutschen Literatur. Hrsg. von Jörg Krappmann und Jaromír Czmero. Olomouc: Palacký-Universität Olomouc.

Anežka Klimentová / 491179@mail.muni.cz

Masarykova univerzita, Filozofická fakulta, Ústav germanistiky, nordistiky a nederlandistiky, Arna Nováka 1, 60200 Brno, CZ 